osteoporosis was found in 22 patients (55\%), osteopenia - in $8(20 \%)$, and norm - in $4(10 \%)$ patients. The BMD values $\left(\mathrm{g} / \mathrm{cm}^{2}\right)$ in the group were as follows:

\begin{tabular}{lllll}
\hline & & & \multicolumn{2}{c}{ Percentiles } \\
\cline { 4 - 5 } & $\mathrm{N}$ & Mean & 25 th & 75th \\
\hline BMD L1-L4 & 40 & 1,06 & 0,88 & 1,25 \\
BMD Prox.Femur & 32 & 0,56 & 0,61 & 0,81 \\
BMD Forearm & 34 & 0,48 & 0,43 & 0,54 \\
\hline
\end{tabular}

Peripheral bone fractures were diagnosed in $15(32.6 \%)$ patients -9 men and 6 women; $25(62.5 \%)$ patients had no fractures. For the first time, fractures were reported in patients aged from 33 to 69 years (mean 55.9 \pm 9.5 ). The localization of fractures was as follows: femur - in 8 patients $(20 \%)$, forearm - in $6(15 \%)$, shin bones - in $1(2.5 \%)$ patients. Despite lower BMD rates in women, there were no significant differences in the frequency of fractures depending on sex. Correlation analysis (for Spearman) showed the relationship of fractures with age $(r=-0.31, p<0.05)$, femur BMD in general $(r=-0.53, p<0.01)$ and forearm BMD $(r=-0.44, p<0.01)$.

Conclusion: There is a high incidence of osteoporosis, mainly in the proximal femur and forearm in patients of the older age group with AKU. In the lumbar spine (due to the development of calcification of the intervertebral discs and ligamentous apparatus), osteoporosis is rarely detected, but the frequency of osteopenia is quite high. $32.6 \%$ patients had a history of skeletal bone fractures, and the sex of the patients did not affect the risk of fractures. The occurrence of fractures in patients with AKU is associated with low BMD values of the proximal femur.

Disclosure of Interests: None declared

DOI: 10.1136/annrheumdis-2020-eular.6380

\section{AB1043 AWARENESS OF THYROID EYE DISEASE, AN AUTOIMMUNE CONDITION, AMONG RHEUMATOLOGISTS}

B. Lamoreaux ${ }^{1}$, M. Francis-Sedlak ${ }^{1}$, R. Holt ${ }^{1}$, J. Rosenbaum ${ }^{2} .{ }^{1}$ Horizon Therapeutics, Lake Forest, United States of America; ${ }^{2}$ Oregon Health \& Science University, Ophthlamology, Portland, United States of America

Background: Autoimmune inflammatory conditions of the eye may be associated with rheumatic diseases such as rheumatoid arthritis, systemic lupus erythematosus, and granulomatosis with polyangiitis. This is also observed with thyroid eye disease (TED). Loss of immune tolerance to the thyroid stimulating hormone receptor has thyroidal consequences and nearly $40 \%$ of patients with Graves' disease also have clinically evident Graves' orbitopathy or TED. ${ }^{1}$ TED results from tissue inflammation that causes retro orbital fat expansion ${ }^{2}$ and extraocular muscle enlargement ${ }^{2}$ and stiffening. ${ }^{3}$ Because the orbital cavity is bony and of limited volume, proptosis and, in severe cases, optic nerve compression, can result. In many patients, muscle changes also cause ocular motility issues and double-vision. Because TED can have a similar presentation to other inflammatory orbital diseases (e.g., granulomatosis with polyangiitis) and Graves' disease patients frequently have other autoimmune conditions (10\% of Graves's patients also have rheumatoid arthritis), ${ }^{4}$ rheumatologists are likely to care for, or even diagnose, patients with TED.

Objectives: This analysis sought to understand rheumatologists' knowledge, and degree of participation in the treatment, of TED including referral patterns from ophthalmologists and endocrinologists for infusion therapies.

Methods: Rheumatologists practicing in the United States attended an educational session and agreed to complete a 12-item survey regarding TED awareness, referral patterns, and management.

Results: Of the 47 rheumatologists surveyed, 45 (96\%) were familiar with TED. Ten $(21 \%)$ physicians reported managing patients with TED, but the majority of physicians (62\%) reported that they co-managed other autoimmune diseases in patients who also had TED. Additionally, $98 \%$ and $64 \%$ of polled rheumatologists had received referrals from ophthalmologists and endocrinologists, respectively, for autoimmune disease management or infusion therapy. Ophthalmology referrals for intravenous (IV) medication administration were most frequently for biologics (82\%), but some referrals were also made for corticosteroids $(2 \%)$ or other medication (13\%) infusions. Only $23 \%$ of rheumatologists had administered a biologic specifically for TED (rituximab: $17 \%$, tocilizumab: $2 \%$, other: $4 \%$ ), but $89 \%$ expressed an interest in administering a TED-specific monoclonal antibody therapy, awaiting FDA approval.

Conclusion: Nearly all surveyed rheumatologists were aware of the signs and symptoms of TED, although most did not actively manage or administer medication for TED. Given the high level of interest in infusing novel, TED-specific biologics, rheumatologists may become an integral part of TED patient management with the approval of a new biologic, teprotumumab, for thyroid eye disease.

\section{References:}

[1] Bartley GB, et al. Am J Ophthalmol 1996;121:284-90.

[2] Forbes G, et al. AJNR Am J Neuroradiol 1986;7:651-656.

[3] Simonsz HJ, et al. Strabismus 1994;2:197-218.

[4] Cardenas Roldan J, et al. Arthritis 2012 2012;864907.

Disclosure of Interests: Brian LaMoreaux Shareholder of: Horizon Therapeutics, Employee of: Horizon Therapeutics, Megan Francis-Sedlak Shareholder of: Horizon Therapeutics, Employee of: Horizon Therapeutics, Robert Holt Shareholder of: Horizon Therapeutics, Employee of: Horizon Therapeutics, James Rosenbaum Consultant of: AbbVie, Corvus, Eyevensys, Gilead, Novartis, Janssen, Roche, UCB Pharma; royalties from UpToDate

DOI: 10.1136/annrheumdis-2020-eular.2049

\section{AB1044 CLINICAL AND IMMUNOLOGICAL FEATURES OF A SERIES OF PATIENTS WITH RHUPUS}

F. Lozano Morillo ${ }^{1}$, T. Almorza ${ }^{1}$, P. Lavilla ${ }^{1}$, M. Retuerto-Guerrero ${ }^{1}$, B. A. Blanco Cáceres ${ }^{2} .{ }^{1}$ Hospital Universitario 12 de Octubre, Madrid, Spain; ${ }^{2}$ Hospital Universitario Ramón y Cajal, Madrid, Spain

Background: Since its first description in 1971 by Schur, many authors have discussed whether rhupus is an overlap syndrome between RA and SLE, a particular form of SLE with prominent and frequently erosive joint involvement,or if it is a distinct clinical and immunological entity. There are several published case series in medical literature describing the features of that uncommon syndrome that constitutes about $0.01-2 \%$ of all systemic rheumatic diseases.

Objectives: To describe demographic, clinical and immunological features of a series of patients with rhupus syndrome and to compare them with previously reported series in the literature.

Methods: Review of clinical records of patients attended in a Tertiary Care Rheumatology Unit that fulfil classification criteria for RA (either ACR 1987 or ACR/EULAR 2010) and SLE (either ACR 1997 or SLICC 2012). In addition, a manual search of patients with positivity for both anti-CCP (defined as $>3 \mathrm{UI}$ / $\mathrm{mL}$ ) and specific SLE antibodies (either anti-DNAds by IIF+- or anti-Sm by multiplex assay) was conducted. We excluded patients with known mixed connective tissue disease, drug-induced SLE as well as RA patients with anti-DNAds+ or anti-Sm+ without clinical features of SLE.

Results: We identified 8 patients, all of them women (4 of Latin American origin, 3 Caucasians and 1 Arab) with a mean age at diagnosis of 35 years (range:19-63 years) and a mean duration of disease of 9 years $( \pm 10.5$ years) RA and SLE were diagnosed simultaneously in $50 \%$ of cases $(37.5 \%$ onset as RA and $12.5 \%$ as SLE, being the mean time between both diagnoses of 16.5 months in those cases). Immunological features of patients are summarized in Table 1. An erosive form of arthritis is present in $37.5 \%$. As extra-articular involvement, $75 \%$ have skin lesions (photosensitivity, malar rash, oral ulcers and alopecia as major features) and 100\% haematological alterations with Iymphopenia (37.5\% thrombopenia). Serositis (37.5\%), renal (25\% biopsy-proven lupus nephritis, $12.5 \%$ non-nephrotic proteinuria) and neurological (present only in one patient) involvement were less common findings. Most common therapies in our series were glucocorticoids $(100 \%$ of cases, with a mean dose of $21.25 \pm 13.5 \mathrm{mg} /$ day at onset), antimalarials $(87.5 \%)$ and methotrexate $(87.5 \%) .50 \%$ of patients required biologic therapy (2 etanercept, 1 adalimumab, 1 rituximab) for inadequate disease control with conventional synthetic DMARDs.

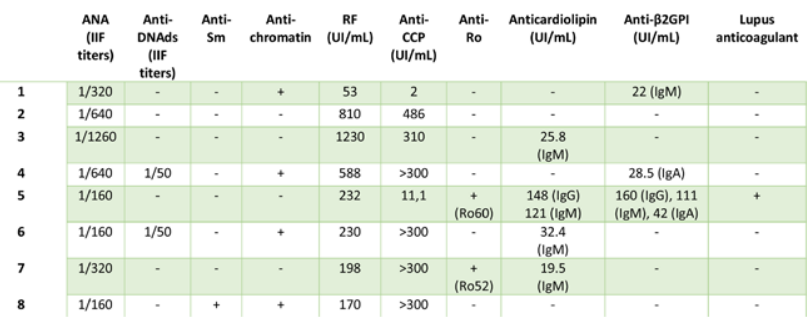

Conclusion: Prevalence of erosive arthritis in our patients is lower than previously reported, though as a limitation an imaging technique with a higher sensitivity for erosion detection than simple X-ray (such as US or MRI) was not available. Moreover, our series sample is small considering the low prevalence of this entity. The proportion of patients with simultaneous diagnosis of both RA and SLE is also higher (with a shorter interval between both diagnoses when this is not the case), so it is the proportion of patients receiving biologic therapy. The 
rest of clinical and immunological features were similar to previously described in other series.

References:

[1] Amezcua Guerra LM. Overlap between systemic lupus erythematosus and rheumatoid arthritis: is it real or just an illusion? J Rheumatol 2009; 36: 4-6.

[2] Li J, Wu H, Huang X, Xu D, Zheng W, Zhao Y, et al. Clinical analysis of 56 patients with rhupus syndrome: manifestations and comparisons with systemic lupus erythematosus. Medicine 2014; 93(10).

[3] Simón JA, Granados J, Cabiedes J, Ruiz Morales J, Alcocer Varela J. Clinical and immunogenetic characterization of Mexican patients with rhupus. Lupus 2002; 11: 287-292

[4] Tani C, D’Aniello D, Delle Sedie A, Carli L, Cagnoni M, Possemato N, et al. Rhupus syndrome: assessment of its prevalence and its clinical and instrumental characteristics in a prospective cohort of 103 SLE patients. Autoimmun Rev 2013; 12: 537-541.

Disclosure of Interests: None declared

DOI: 10.1136/annrheumdis-2020-eular.4972

\section{AB1045 $\quad$ CLINICAL, ANALYTICAL AND RADIOLOGICAL CHARACTERISTICS OF A COHORT OF PATIENTS WITH SARCOIDOSIS.}

I. Madroñal García ${ }^{1}$, C. Aguilera $\operatorname{Cros}^{1}$, L. Mendez Diaz ${ }^{1}{ }^{1}$ Hospital Universitario Virgen del Rocío, Reumatologia, Sevilla, Spain

Background: Sarcoidosis is a systemic disease whose etiology is unknown. It is characterized by the formation of granulomas in any tissue of the organism. Ganglionic, pulmonary and cutaneus involvement is the most prevalent. Objectives:

1. Describe clinical characteristics of a cohort of patients with sarcoidosis diagnosed.

2. Define the association between the ACE's number at diagnosis, radiological lung stage, treatment and course of disease.

3. Evaluate if the extrapulmonary involvement is related to the course of the disease.

Methods: Descriptive retrospective study of patients with $\mathrm{S}$ diagnosis treated in our Hospital in 2019. Data were obtained by reviewing medical records. Chisquare tests and parametric tests have been used to establish the differences described in the objectives.

Results: 102 patients diagnosed with sarcoidosis have been included, (51\% females) with an average age of $56 \pm 11$ years. Suspected diagnosis at the onse of disease was S in $70.6 \%$ of patients, followed by suspected lymphoma $(20.6 \%)$ The average time for the definitive diagnosis of $S$ was 9.5 months. $70.6 \%$ of the patients had elevated ACE titles at the beginning. Regarding the clinical manifestations, $18.6 \%$ of the patients presented fever at the beginning and $66.7 \%$ extrathoracic clinical manifestations. $72.5 \%$ have lymph node adenopathies, and in $91 \%$ there is thoracic involvement (the most frequent pulmonary stage is stage II). A biopsy was performed in $84.3 \%$ of the patients, the lung biopsy being the most performed (52.3\%). $88.2 \%$ of patients received corticosteroid treatment at the onset of the disease (currently under treatment with corticosteroids $37.3 \%$ ). $50 \%$ of patients are treated with immunosuppressants, Methotrexate was the most used. 5 patients are treated with biological therapy (AntiTNF).

Regarding the course of the disease, $51 \%$ of the patients have a chronic course $45.1 \%$ are in remission and $3.9 \%$ have suffered a relapse of the disease. In this study, no significant relationship was found between the ACE values at the onset of the disease, the pulmonary stage and the course of the disease.

According to our data, patients presenting with extrathoracic clinical manifestations need more frequently corticosteroid treatment $(p=0.017)$ and immunosuppressive treatment $(p=0.001)$ with respect to patients who do not have an extrathoracic clinic. In addition, patients with an extrathoracic clinic present more frequently a chronic course of the disease than those who do not $(p=0.019)$

Conclusion: The results described in this study are similar to those found in the literature. The differences found can be explained because patients presenting with extrathoracic clinical manifestations have a more complicated management and need more treatment than those with only pulmonary involvement, even patients with radiological stage I do not usually need treatment, only surveillance.

Disclosure of Interests: None declared

DOI: 10.1136/annrheumdis-2020-eular.6414

\section{AB1046 JOINT MANIFESTATIONS IN A COHORT OF PATIENTS WITH SARCOIDOSIS IN A THIRD LEVEL HOSPITAL.}

I. Madroñal García ${ }^{1}$, C. Aguilera $\operatorname{Cros}^{1}$, L. Mendez Diaz ${ }^{1}{ }^{1}$ Hospital Universitario Virgen del Rocio, Reumatologia, Sevilla, Spain
Background: Sarcoidosis (S) is a systemic granulomatous disease of unknown etiology, which most frequently affects the ganglion, lung and skin, although it can affect other organs, including the musculoskeletal system.

Objectives:

Describe the clinical, analytical and radiological characteristics of patients diagnosed with $\mathrm{S}$ presenting joint manifestations.

To assess the association between patients who have joint manifestations and the use of corticosteroids (C) and immunosuppressants (IS), with respect to those without joint involvement.

Methods: Retrospective descriptive study of patients with diagnosis of $\mathrm{S}$ with joint manifestations, treated in our Hospital from 2017 to 2019. Data were obtained by reviewing medical records. Chi square tests and Fisher's exact test have been performed to establish the differences described in the objectives.

Results: From a database of 102 patients with S, 18 presented joint manifestations ( $50 \%$ women), with a mean age of $57 \pm 6$ years. Of these patients, 4 $(22.2 \%)$ have presented positive ANA. Regarding the clinic, 3 patients presented the association of polyarthritis and bilateral ankle swelling, 8 patients presented with polyarthritis, 3 monoatritis and 4 patients presented bilateral ankle swelling $61.1 \%$ had fever at the onset of the disease.

14 patients $(77.8 \%)$ had high ACE values at the onset of the disease, without presenting significant differences with respect to all patients diagnosed with $S$ who do not have joint involvement.

All patients received treatment with $\mathrm{C}$ and 10 patients $(55.5 \%)$ needed an IS treatment, finding no differences with respect to patients who do not have joint involvement $(p=0.92)$

On the course of the disease, the majority of patients with joint involvement have a chronic course $(72.2 \%)$. Nor were significant differences found when compared with patients who have no joint involvement $(p=0.73)$.

Conclusion: Patients with joint involvement in our study have been $17.6 \%(18)$ an approximate result to that described in the literature (over $10 \%$ ), although our result may be increased by the fact that the patients who are followed in Rheumatology present or have presented joint involvement. No significant differences were found between patients with $\mathrm{S}$ who presented joint involvement and those who did not, with respect to the initial ACE values, treatment and disease course. Prospective, multicenter and larger sample studies are necessary to better understand these associations.

Disclosure of Interests: None declared

DOI: 10.1136/annrheumdis-2020-eular.6444

\section{AB1047 \\ IGG4-RELATED DISEASE PRESENTATION REQUIRES ADMISSION TO THE EMERGENCY DEPARTMENT IN THE MAJORITY OF CASES}

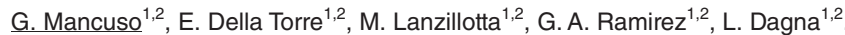
${ }^{1}$ Università Vita-Salute San Raffaele, Milano, Italy; ${ }^{2}$ San Rafael Hospital, Unit of Immunology, Rheumatology, Allergy and Rare Diseases, Milano, Italy

Background: IgG4-related disease (IgG4-RD) is generally considered a chronic fibro-inflammatory condition with insidious presentation and subclinical course. Our clinical experience, however, suggests that a sizable proportion of patients experience multiple accesses to the emergency department (ED), either at dis ease onset or during the disease course.

Objectives: In the present study we aimed (i) to assess the prevalence of acute manifestations of IgG4-RD at disease onset requiring referral to the $E D$, and (ii) to calculate the diagnostic delay from the initial acute presentation.

Methods: We revised our database and identified patients admitted to the ED because of symptoms lately attributed to IgG4-RD onset (Group 1) and those that were referred to our outpatient clinic without previous urgent manifestations (Group 2). Acute manifestations were clustered based on the anatomical distric affected by IgG4-RD. Epidemiological, clinical, and serological features of Group 1 and Group 2 were compared.

Results: The study included 141 patients with IgG4-RD. 76 (54\%) presented to the ED at disease onset. The most common clinical manifestations requiring admission to the ED were jaundice (53\%), abdominal pain $(41 \%)$, and feve (10\%). Gastrointestinal involvement was the most frequent cause of referral to the ED ( $71 \%$ of cases), followed by involvement of the retroperitoneum $(14.5 \%)$, and of the nervous system (6.6\%). Pancreato-biliary involvement was significantly more frequent in Group 1. Head, neck, salivary and lacrimal gland involvement was more frequent in Group 2. The diagnostic delay was significantly shorter in Group 1 than in

Group 2.

Conclusion: Clinical manifestations associated with IgG4-RD onset require referral to the ED in the majority of cases. This finding contrasts with the genera view of IgG4-RD as a condition with non-acute presentation. 